\title{
Sensitivity analysis to compute advanced stochastic problems in uncertain and complex electromagnetic environments
}

\author{
Sébastien Lalléchère ${ }^{1,2}$, Basile Jannet ${ }^{1,2,3}$, Pierre Bonnet $^{1,2}$, and Françoise Paladian ${ }^{1,2}$ \\ ${ }^{1}$ Clermont University, University Blaise Pascal, Institute Pascal, F-63000 Clermont-Ferrand \\ ${ }^{2}$ CNRS, UMR 6602, IP, F-63171 Aubière \\ ${ }^{3}$ CEA, DAM, Gramat, F-46500 Gramat \\ *corresponding author, E-mail: sebastien. lallechere@univ-bpclermont.fr
}

\begin{abstract}
This paper deals with the advanced integration of uncertainties in electromagnetic interferences (EMI) and electromagnetic compatibility (EMC) problems. In this context, the Monte Carlo formalism may provide a reliable reference to proceed to statistical assessments. After all, other less expensive and efficient techniques have been implemented more recently (the unscented transform and stochastic collocation methods for instance) and will be illustrated through uncertain EMC problems. Finally, we will present how the use of sensitivity analysis techniques may offer an efficient complement to rough statistical or stochastic studies.
\end{abstract}

\section{Introduction}

A large set of electromagnetic (EM) problems are currently treated with a very high level of accuracy from numerical and/or experimental devices. Their deterministic design may reveal quite convenient if input data are precisely controlled. Due to the disturbed EM environment where most of the radio systems evolves, the electromagnetic compatibility (EMC) issues are rarely entirely known: from the number of parameters impinging both the sources, victims and/or coupling paths, the deterministic design of the problem appears utopian. In this context, a crucial point relies on our ability to estimate the impact of different Random Variables (RV) on given outputs.

\subsection{Stochastic context}

The Monte Carlo (MC) formalism remains one of the most spread statistical technique to handle with stochastic treatments. As explained in [1], the MC method needs few requirements on the chosen random output. In an integration approach, this technique is well suited for singular (irregular) kernels and it is not compulsory to access analytic information on their statistical form. The "classical" MC reveals independent of the problem dimension and can be used for many stochastic issues involving a high numbers of RV. Nevertheless, as pointed out in [2], the main disadvantage of $\mathrm{MC}$ remains its slow convergence rate. Thus, it may appear computationally prohibitive (even for highdimensional problems) but is still interesting to access a "reference" result. Finally, the difficulty remains to set the threshold defining the MC convergence level.

Different stochastic techniques have been successfully computed since 2002 in order to give a more realistic view of EM simulations including uncertainties. We may for instance cite the unscented transform (UT) $[3,4,5]$ or the "Lagrange" stochastic collocation (SC) $[6,7,8,9,10]$ methods, the kriging technique [11], the polynomial chaos expansion [12, 13, 14], and the experimental design [15]. Far from appearing as an exhaustive review of stochastic and statistical methods in electromagnetism, the previous quoted techniques were mainly applied to numerical simulations including various EM domains: shielding effectiveness $[3,4,5,9]$, scattering and propagation $[6,9]$, susceptibility $[9,10,11,15]$ and/or (bio-)EMC/EMI $[7,8,12,13,14]$ problems.

\subsection{Aims and motivations}

From domains as environmental modeling, mechanics, safety and reliability, the sensitivity analysis (SA) methods are widely spread from twenty years. We may commonly define the SA for a given physical model as a method used to determine how various values of an independent variable will impact a particular dependent variable under a given set of assumptions. This is typically a deterministic view regarding a mathematical expression as a function of "inputs" that produce "outputs"; deterministic means that the same set of "input" variables gives always the same "output" values. For a considered "output" quantity, the goal is to determine the contributions from "input" and their interactions with the model.

Various objectives are expected from SA: modelling complex and combined phenomena, calibrating "input" parameters, simplifying a model (decreasing the number of variables), organizing the "input" parameters both from qualitative and quantitative points of view. Obviously, this treatment may lead to a better understanding of the studied system, to simplify it and to check its reliability.

Different techniques are available, and we may mention, without exhaustiveness the screening techniques (to access to the most influential inputs among a large number from coarse sorting), and the variance based sensitivity indices (measures of importance). Thus, both qualitative and 
quantitative treatments are allowed chosing one of the previous techniques. Another way to present SA methods may be to consider if the sensitivity needs require a local or a global analysis. This is equivalent to consider respectively the linearity hypothesis between "inputs" or to study the interactions between parameters. In the following, we will focus on global analysis.

\section{Theoretical principles}

This section is dedicated to the theoretical details of the stochastic and SA methods used in this work.

\subsection{Notations and approaches}

In our models, a stochastic parameter $Z$ will be given from a RV $\hat{u}$ as follows:

$$
Z=Z^{0}+\hat{u}
$$

where $Z^{0}$ is called the initial value (mean) and $\hat{u}$ is characterized by a given statistical distribution law (zero-mean with a certain variance). In the following, the random value $Z$ may represent different kind of parameters: geometrical details, material values, and/or source characteristics.

In this paper, we will consider a model for which an "output" $F$ is a deterministic function of $k$ "inputs" denoted by $u_{1}, u_{2}, \ldots u_{k}$. We may sum up the $k$ "inputs" in a row vector $\mathbf{u}$

$$
\mathbf{u}=\left(u_{1}, u_{2}, \ldots, u_{k}\right),
$$

and write $F(\mathbf{u})$ the global effect of $k$ "inputs" a priori considered as RV for a computational (analytical and/or numerical) model $F$.

The aim of this study is to focus on two efficient methods: the UT [3] and the SC [9] techniques. These two previous approaches rely on the same philosophy with the simplicity of MC but faster convergence rates. Their main advantage remains their high precision.

A sensitivity analysis technique widely spread in models of large dimension is the screening design proposed in [16]. It deals efficiently with models containing a lot of input factors and may lead to quick qualitative assessment since the number of model evaluations is linear in the number of model factors. Obviously other techniques may be used with different degrees of accuracy and efficiency to rank RV by influence.

\subsection{Stochastic methods foundations}

In the following, we will detail theoretical principles considering 1-RV formalism. We may extend it to multi-RV cases without any loss of generality.

\subsubsection{UT basis}

As explained in [3], the use of the UT method is similar to the MC technique. The main difference relies on the number of realizations needed to obtain the statistical moments of a given output. Thus, instead of several thousands of repetitions, only a few selected ones are necessary.

Some conditions are required to compute UT for a single RV: we may know both the moments of the RV $\hat{u}$ and the nonlinear mapping of the random output $(I(\hat{u}))$. Its $n$th order moment may be expressed as follows

$$
\mathbb{E}\left\{I(\hat{u})^{n}\right\}=\int I(u)^{n} p d f(u) d u,
$$

where $p d f(\mathrm{u})$ is the probability density function of the RV $\hat{u}$. A discrete equivalent of the relation (3) is used for the integration

$$
\int I(u)^{n} p d f(u) d u \approx \sum_{i} \omega_{i} I\left(S_{i}\right)^{n},
$$

where $S_{i}$ are the so-called sigma points (for the integration). If the nonlinear mapping $I(\hat{u})$ is well behaved, it could be expressed from Taylor polynomial series $\left(g_{j}\right.$ coefficients) as

$$
\int I(u)^{n} p d f(u) d u=\sum_{j=0}^{\infty} g_{j} \int u^{j} p d f(u) d u .
$$

From the discrete sum (4), each integration term of (5) may be expressed from $k+1(k=0,1,2, \ldots)$ equations as

$$
\int u^{k} p d f(u) d u \approx \sum_{i} \omega_{i} S_{i}^{k}=\mathbb{E}\left\{\hat{u}^{k}\right\} .
$$

The nonlinear system depicted in (6) allows the computation of the sigma points $S_{i}$ and weights $\omega_{i}$ from the moments of the RV $\hat{u}$. As detailed in [3], the minimum number of $S_{i}$ points for a given order of the UT technique may be derived using the Gauss quadrature schemes. Indeed, considering (6), the solution is not unique and different sets of $\left(S_{i}, \omega_{i}\right)$ may be obtained as illustrated in the following.

\subsubsection{SC foundations}

The idea of the technique is to choose a polynomial approximation of a given output $I$ depending on a random parameter $Z$ (1). In a first time, the function $S \rightarrow I\left(Z^{0} ; S\right)$ is expanded in Lagrangian basis functions of order $n$

$$
I\left(Z^{0} ; S\right) \approx \sum_{i=0}^{n} I_{i}\left(Z^{0}\right) L_{i}(S)
$$

with $L_{i}(S)=\prod_{\substack{j=0 \\ j \neq i}}^{n} \frac{S-S_{j}}{S_{i}-S_{j}}$. One of the most interesting properties of the Lagrangian basis relies on its reducing characteristic: $L_{i}\left(S_{j}\right)=\delta_{i j}$ (Kronecker $\delta$ ) and we may write $I_{i}\left(Z^{0}\right)=I\left(Z^{0} ; S_{i}\right)$. Then, the integration computation is based upon the Gauss quadrature with identical points $S_{i}$ than the ones previously needed by the UT method

$$
\int_{D} p d f(u) f(u) d u \approx \sum_{i=0}^{n} \omega_{i} f\left(S_{i}\right)
$$


where $p d f$ is the probability density function introduced in the previous section. Similarly to the UT case, the real numbers $\omega_{i}$ are called integration weights. From (7), we may detail $I$ with its polynomial approximation

$$
\mathbb{E}\left\{I\left(Z^{0} ; S\right)\right\}=\sum_{i=0}^{n} I_{i}\left(Z^{0}\right) \int_{D} L_{i}(s) p d f(s) d s
$$

From (9), we may straightforward compute weights following

$$
\omega_{i}=\int_{D} L_{i}(s) p d f(s) d s .
$$

We will detail in the following the statistical moments computation enabled by the pair $\left(S_{i} ; \omega_{i}\right)$.

\subsubsection{Multi-RV cases}

Based upon the results obtained for a single RV, the UT Taylor polynomial representation is still suitable for two RV. In the two variables case, the system (6) may be written using statistical moments cross terms [5] following

$$
\sum_{i} \omega_{i}\left(S_{i}^{1}\right)^{m}\left(S_{i}^{2}\right)^{n}=\mathbb{E}\left\{\hat{u}_{1}^{m} \hat{u}_{2}^{n}\right\} .
$$

The sigma points and weights are computed for the two RV $\hat{u}_{1}$ and $\hat{u}_{2}$ and derived from the possible power combinations of $m$ and $n$ (natural numbers) with $0 \leq m+n \leq 4$. Once more there are several possible solutions: in the following we will give different sets of sigma points and weights solving the system (11).

Regarding the SC computing, the previous theoretical elements may also be generalized to the multivariate case. Therefore, by considering a two-variable random problem, for instance involving two RV $\hat{u}_{1}$ and $\hat{u}_{2}$ standing for two random parameters respectively $Y$ and $Z$, we may write $Y$ and $Z$ from the relation (1) including two initial values $\left(Y^{0}\right.$ and $\left.Z^{0}\right)$. From the same theoretical foundations, we may project the function $(s, t) \rightarrow I\left(Y^{0}, Z^{0} ; s, t\right)$ on a Lagrangian basis

$$
I\left(Y^{0}, Z^{0} ; s, t\right) \approx \sum_{i=0}^{n} \sum_{j=0}^{n} I_{i j}\left(Y^{0}, Z^{0}\right) L_{i}(s) L_{j}(t),
$$

with $I_{i j}\left(Y^{0}, Z^{0}\right)=I\left(Y^{0}, Z^{0} ; s_{i}, t_{j}\right)$. It is rather simple from (12) to compute the moments of the output $I$ through a tensor product in each direction (i.e., for each $\mathrm{RV}$ ) based upon the generalization of (8).

Finally, in a further section one may find the details of the statistical moments computation for a single RV (Table 1). The principle remains quite the same both for UT and SC in multivariate case since a new set of points/weights are defined. As illustrated in Fig. 1, even if the SC technique may appear heavier than the UT method (tensor product), it presents two main advantages. Indeed, the computation of new sigma points/weights sets is rather simple and the efficiency/accuracy of the results remain particularly interesting. The Fig. 1 presents sigma/weight points obtained from a standard normal distribution from two UT sets and one SC computation.

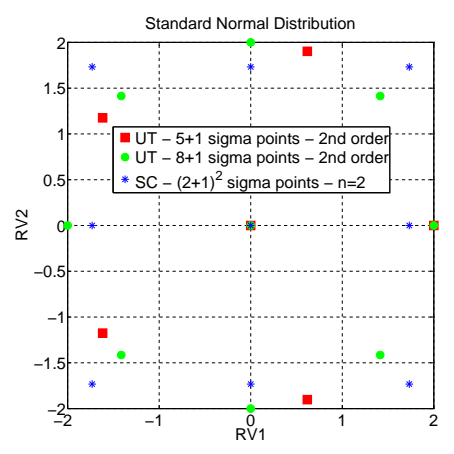

Figure 1: Different sigma points distributions for two RV (UT and SC).

\subsubsection{Computing the sigma points/weights and the statis- tical moments}

Although the UT and SC appear very similar considering the computation of their respective sigma points/weights, they differ from their basis. From the different solutions proposed for the one-variable case, the minimum number of pairs $\left(S_{i}, \omega_{i}\right)$, for a given order $n$, is straightforward available by the Gauss quadrature scheme (identical to the SC case). Therefore, the expression of the integration points/weights is similar for the UT [3] and SC [8], and the results will be identical.

The Tab. 1 gives an overview of the points/weights in single RV case following a standard normal distribution. We may construct similarly the multi-RV set of points/weights for UT and SC. Based upon [5], it is possible to extend the previous set of points (Tab. 1) to the numerical examples presented in the following (two RV) including the variances of the distribution laws. As explained previously (Fig. 1), the UT solution is not unique when solving the system (11) and we may obtain (for a same order) different sets of sigma/weights points.

Table 1: Sigma/weights pairs $\left(S_{i}, \omega_{i}\right)$ for $1-\mathrm{RV}$ case with a standard normal law (UT/SC).

\begin{tabular}{c|c|c|c|c|c|c}
\hline \multicolumn{2}{c|}{$n$} & Pt1 & Pt2 & Pt3 & Pt4 & Pt5 \\
\hline \multirow{2}{*}{2} & $S_{i}$ & $-\sqrt{3}$ & 0 & $\sqrt{3}$ & $\mathrm{x}$ & $\mathrm{x}$ \\
& $\omega_{i}$ & $1 / 6$ & $2 / 3$ & $1 / 6$ & $\mathrm{x}$ & $\mathrm{x}$ \\
\hline \multirow{2}{*}{4} & $S_{i}$ & -2.857 & -1.356 & 0 & 1.356 & 2.857 \\
& $\omega_{i}$ & 0.011 & 0.222 & 0.533 & 0.222 & 0.011 \\
\hline
\end{tabular}

In order to illustrate the multivariate computing of the $\left(S_{i} ; \omega_{i}\right)$ sets, we may focus on a bidimensional RV case, $R V 1+R V 2$ (see Fig. 1) where $R V 1$ and $R V 2$ follow a standard normal distribution $(\mathbb{N}(0 ; 1))$.

The collocation technique gives the collocation points $\left(S_{i}\right)$ and weights $\left(\omega_{i}\right)$ necessary to compute, for instance, the 1 st and 2nd-order statistical moments of the $F$-mapping given in section 2.1 .

From previous notations $(\mathbb{E}(F)$ for instance), in order to simplify the discussion, the $\langle F\rangle$ and $\sigma_{F}^{2}$ symbols will stand respectively for the mean and variance of $F$. 
Table 2: UT/SC computation of statistical moments (1st and 2nd) for the 1-RV case.

\begin{tabular}{c|c}
\hline Stat. moment & Computation \\
\hline Mean & $\operatorname{mean}(F)=\sum_{i=0}^{n} \omega_{i} F_{i}$ \\
Variance & $\operatorname{var}(F)=\sum_{i=0}^{n} \omega_{i} F_{i}^{2}-[\operatorname{mean}(F)]^{2}$ \\
\hline
\end{tabular}

The formalism detailled in Tab. 2 is common to the UT and SC methods for single RV. The computation of the sigma points and weights (multi-RV) may be found respectively in $[5,8]$. In the following, we will present the numerical differences existing between the UT and SC multivariate results.

\subsection{Sensitivity analysis}

In early 90's, an approach was proposed by Morris [16] to discriminate the "inputs" of a given modelling (for instance a determined computational relation $F$ ). This work aimed to determine which "inputs" had important effects on a given "output". This model is widely used since it is easy to compute and may reveal particularly efficient with models containing hundreds of "inputs". Thus, among the numerous methods available to conduct SA, we use in this paper the method introduced by Morris [16] (see [17, 18] for a review of SA techniques).

The main idea from [16] was, among a large variety of "inputs", to reasonably compute which may be considered negligible, additive and linear, non-linear and/or in interactions with other "inputs". With this objective, a "discretized" approach was originally proposed by Morris. Indeed, the factorial sampling plan proposed in [16] is composed of individual randomizations named "one factor-at-atime". The elementary influence of the $i t h$ "input" is computed for a discrete number of values called "level" (given by the $i t h$ factor varying range). Let $\mathbf{u}$ be a given RV vector containing $k$ random "inputs" as depicted in the relation (2). We may set a varying range depicted by their minima and maxima values for each RV respectively $u_{1}^{\min }, u_{1}^{\max }$, $\ldots, u_{k}^{\min }, u_{k}^{\max }$ defining the definition domain $\Omega$. Originally, the sensitivity approach requires to sample $p$ levels for each $i t h$ RV through a given $\delta_{i}$ parameter. From [16], the $k$ "inputs" were given over $[0 ; 1]$ range. In this work, we propose an elementary influence criterion $c_{i}$, for a given $\mathbf{u}$ value, with $\delta$ a predetermined multiple of $\delta_{i}$

$c_{i}(\mathbf{u})=\frac{F\left(u_{1} \ldots u_{i-1}, u_{i}+\delta, u_{i+1} \ldots u_{k}\right)-F(\mathbf{u})}{\delta} \times \chi_{i}$,

where $\chi_{i}$ is a given normalization coefficient depending on the $\left[u_{i}^{\min } ; u_{i}^{\max }\right]$ range. In the following, $D_{i}$ will stand for the finite distribution of elementary influence criterion $c_{i}(i=1, \ldots, k)$ obtained from randomly sampling a number $n$ of different $\mathbf{u}$ vectors.

Obviously, the choice of $\delta_{i}$ may influence the quantitative results proposed by [16]. Indeed, the number of discrete "levels" $p+1$ given directly represents the sampling sharpness $\left(\delta_{i}=\frac{u_{i}^{\max }-u_{i}^{\min }}{p}\right)$. The principle used in [19] requires to uniformly sample the $k$-dimensional parallelepiped standing for the $\mathbf{u}$ potential values from $\Omega$. Given a number $n$ of starting points, we may define $n$ u-vectors following the simple rule "one-factor-at-time". This means moving from $\mathbf{u}=u_{1}, u_{2}, \ldots, u_{k}$ to $\mathbf{u}+\delta \mathbf{e}_{i}$, where $\mathbf{e}_{i}$ is a zero vector with a unit as its ith component. We may ensure that the "translated" point $\mathbf{u}+\delta \mathbf{e}_{i}$ is still in the definition domain. From an iterative process, we may obtain the discrete $D_{i}$ distribution from the relation (13).

As explained in [19], each RV is evaluated regarding two distinct measures: $\mu_{i}$ and $\sigma_{i}$, being respectively the estimated mean and standard deviation of $D_{i}$. From a practical point of view, it should be better to compute a measure enabling to integrate the potential non-monotonic model behaviour by defining a rougher criterion. Thus, let $\mu_{i}^{*}$ stands for the mean of the distribution $E_{i}$ of the absolute values of $c_{i}$ parameter. $\mu_{i}^{*}$ will be more restrictive than $\mu$ since $D_{i}$ may contain elements contrary signed. They may vanished from the influence estimate and lead to erroneously considered corresponding parameters as negligible. Thus, we may write

$$
\begin{gathered}
\mu_{i}=\frac{1}{n} \sum_{i=1}^{n} c_{i}, \\
\mu_{i}^{*}=\frac{1}{n} \sum_{i=1}^{n}\left|c_{i}\right|, \\
\sigma_{i}=\sqrt{\frac{1}{n} \sum_{i=1}^{n}\left(c_{i}-\mu_{i}\right)^{2} .}
\end{gathered}
$$

Practically, the "Morris" screening design enables to rank RV from a quantitative point of view. It aims to represent the random parameters from a $\left\{\mu_{i}\right.$ (or $\left.\left.\mu_{i}^{*}\right) / \sigma_{i}\right\}$ representation. A high value of " $\mu_{i}$ " reprents an "input" with a high overall impact. A high value of " $\sigma_{i}$ " stands for a parameter highly involved in interaction with other factors. In the following, we will detail results from the $\mu_{i}^{*}$ criterion. Obviously, different parameters from this screening design technique may impact the reliability and efficiency of treatments. Thus, the sampling sharpness may be a crucial point since the grid used to quantify RV elementary effects may be too coarse. In most cases, the discretization grid is set to $p=100$ or 1000 levels. In order to improve the reliability of results, the relations $(14 ; 15)$ are evaluated several times in order to produce a cloud of $N$ points. From a computing efficiency point of view, the "Morris technique" requires $n \times(k+1) \times N$ calls to the computational relation $F$ in order to entirely compute results.

The Morris analysis may be presented throughout an analytical test function $f$ depending on $\mathbf{u}=\left(u_{1}, \ldots, u_{11}\right)$

$$
\begin{aligned}
f(\mathbf{u}) & =u_{1} u_{2}+\frac{5}{u_{3}\left(u_{11}+0.5\right)+\cos \left(u_{4}\right)+0.1} \\
& +4 u_{5}^{3}+4 u_{6}-u_{7}+u_{8} \exp \left(u_{9}\right)+0.2 u_{10}
\end{aligned}
$$




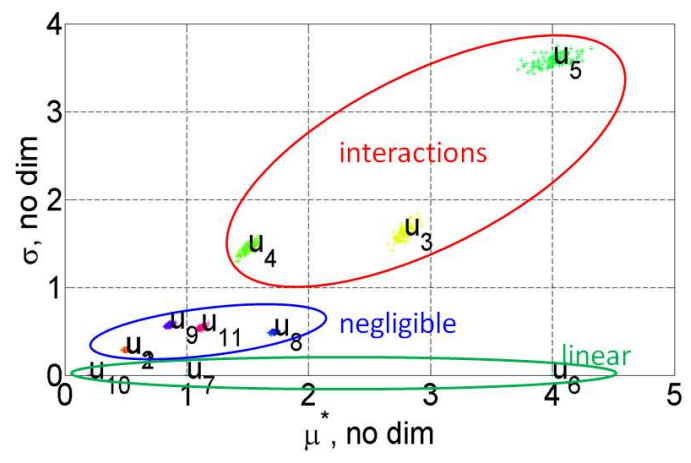

Figure 2: RV ranking from screening design.

with $u_{i}, i=1 \ldots 11 \in[0 ; 1]$. In this case, the initial "Morris" parameters are set following: $n=10, p=1000$ and $N=100$. Thus, the whole process requires 12,000 calls to analytical function $f$ from the relation (17).

As expected comparing relation (17) and Fig. 2, the $u_{6}, u_{7}$ and $u_{10}$ parameters appear as linear ones and may be set apart. Contrary to other values, $u_{i}(i=3,4,5)$ ones may not be neglected (inner interactions inside model). Obviously, their characterization only offer a qualitative approach and it is necessary to proceed to a precise and effective quantitative computation to obtain influence orders.

\section{The stochastic EMC issue}

\subsection{Setup and initial parameters}

In this work, the case of a transmission line illuminated by a plane wave with multiple random parameters is used from [11]: attenuation constant $\alpha$, diameter $d$, frequency $f$, length $L$, height $h$ above an infinite ground plane. The excitation source is a plane wave linearly polarized (Fig. 3). An analytical formulation can be obtained for the current $I$ at load $Z_{L}=1 k \Omega\left(Z_{0}\right.$ is set to $\left.50 \Omega\right)$, and we may write

$$
I=I\left(L, h, d, Z_{L}, E_{0}, \alpha, Z_{0}, f, \theta_{p}, \theta_{e}, \phi_{p}\right)+I_{p},
$$

with $I_{p}$ a randomly distributed disturbing current adding to the formula proposed in [11].

Table 3: Initial sets of parameters for the stochastic study.

\begin{tabular}{ccccccc}
\hline Value & $L(\mathrm{~m})$ & $h(\mathrm{~mm})$ & $d(\mathrm{~mm})$ & $Z_{L}(\Omega)$ & $E_{0}(\mathrm{~V} / \mathrm{m})$ & $\alpha$ \\
\hline Min & 1.2 & 8 & 0.6 & 996 & 996 & 0. \\
Max & 4.5 & 32 & 1.4 & 1004 & 1004 & 0.01 \\
\hline \hline Value & $Z_{0}(\Omega)$ & $f(\mathrm{MHz})$ & $\theta_{p}(\mathrm{rad})$ & $\theta_{e}(\mathrm{rad})$ & $\phi_{p}(\mathrm{rad})$ & $I_{p}(\mathrm{~A})$ \\
\hline Min & 46 & 1 & -0.3 & -0.3 & -0.3 & 0.15 \\
Max & 54 & 50 & +0.3 & +0.3 & +0.3 & 0.25
\end{tabular}

The purpose of adding in (18) the current $I_{p}$ is to input a linear RV into the global model. Even if the problem appears relatively simple, the complexity, here, comes from the number of RV and there is no use facing straightforward this issue considering MC. Obviously, some sparse

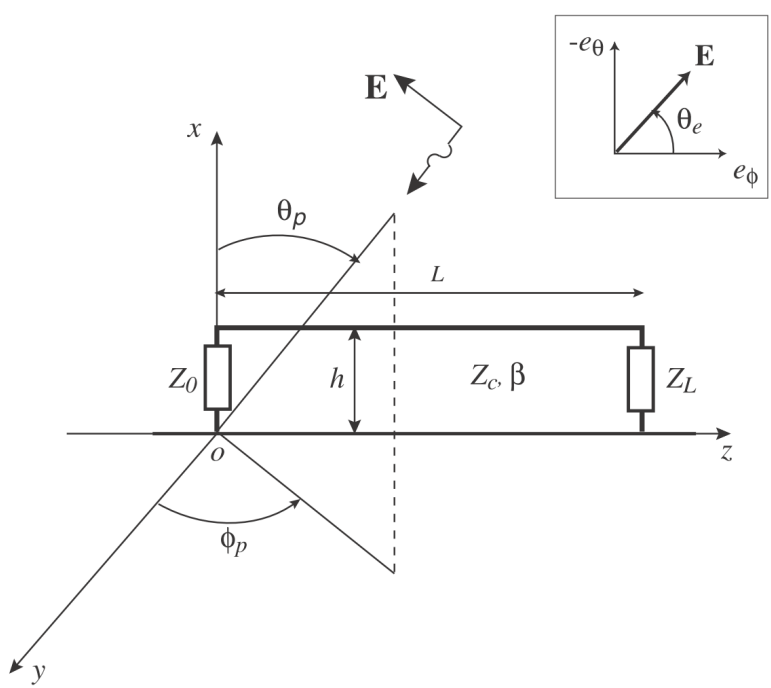

Figure 3: Transmission line illuminated by a plane wave [11].

grid techniques $[12,20]$ may improve considerably the efficiency of stochastic techniques but this may reveal quite restricted if, independently, each RV needs not less than 4 or 5 collocation points. The aim of this study is to propose a two-steps work. First, based upon a qualitative analysis, our aim is to rank the RV from their relative effect and to reduce their total number by setting the least influential parameters to deterministic values. Then, the use of stochastic techniques fits well with a quantitative analysis of the parameters sensitivity.

\subsection{Stochastic techniques accuracy and efficiency}

Since the Taylor polynomial expansion is still usable for two random variables, the UT technique may be used to achieve multivariate stochastic problem. Moreover, as illustrated in [8], the single-variable SC method may be generalized to multi-RV problems. As explained previously, by choosing sigma points from the Gauss quadrature, the UT and SC techniques might appear quite similar with identical integration points and weights. Based upon their distinct foundations, for multivariate case, the different two $\mathrm{RV}\left(S_{i}, \omega_{i}\right)$ sets jointly with the different moments computation involve variations around the numerical results.

In this section, we have arbitrarily chosen the line length $L$ and the source frequency $f$ to achieve a stochastic processing of the EMC problem (Fig. 3). The $L$ and $f$ parameters will be both given by two independent RV (respectively $\hat{u}_{1}$ and $\hat{u}_{2}$ ) following a zero-mean normal distribution respectively with variances $\sigma_{\hat{u}_{1}}^{2}=2.083 .10^{-4}\left(\mathrm{~m}^{2}\right)$ and $\sigma_{\hat{u}_{2}}^{2}=2.083 .10^{10}\left(\mathrm{~Hz}^{2}\right)$. From the expression (1), the two random parameters may be written

$$
\begin{aligned}
L & =L^{0}+\hat{u}_{1} \\
f & =f^{0}+\hat{u}_{2},
\end{aligned}
$$

with $L^{0}$ and $f^{0}$ the length and frequency means. The 


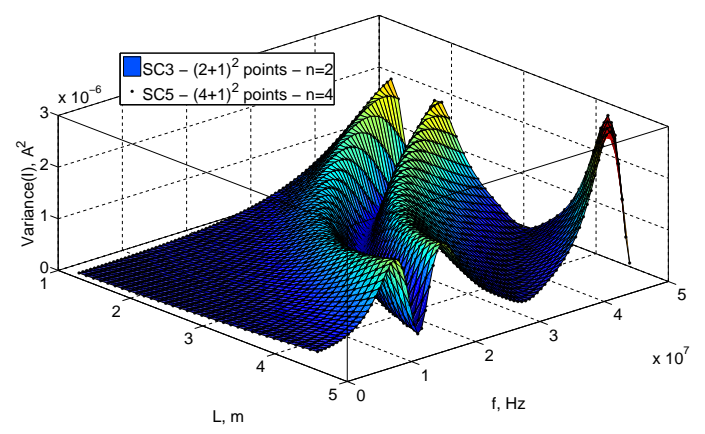

Figure 4: Variance( $(I)$ from SC3 and SC5.

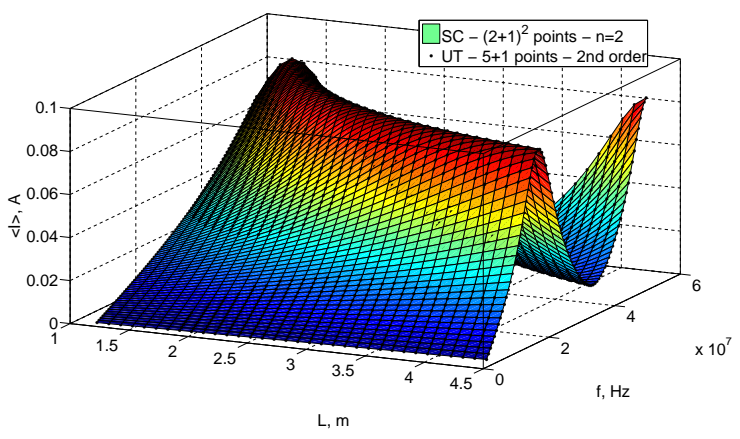

Figure 5: Mean $(I)$ from SC3 and UT5.

Fig. 4 shows how straightforward the current variance may be obtained for a large set of points. The previous random variations are applied to each element of the set $\left(L_{i}^{0}\right.$ $\left.\in D_{L}=[1.2 \mathrm{~m} ; 4.5 \mathrm{~m}] ; f_{j}^{0} \in D_{f}=[1 \mathrm{MHz} ; 50 \mathrm{MHz}]\right)$. The SC convergence and the sensitivity of the model are first showed in Fig. 4. The results depicted in Fig. 4 show the convergence of the SC method (from the $I$ variance). Considering the SC accuracy for $3^{2}$ and $5^{2}$ points (respectively SC3 and SC5), the two data sets almost overlap.

In Fig. 5 and 6, the results from UT fit very well with the "converged" data from SC. Thus, Fig. 5 shows the agreement for $\langle I\rangle$ between SC3 and the approximation from the UT (2nd order) involving $5+1$ points (UT5). A great agreement appears also from Fig. 6 considering the slight differences existing between a same UT accuracy (2nd order) involving $5+1$ or $8+1$ points (respectively UT5 or UT8). The UT5 and UT8 differences rely on the non-uniqueness of the solution as detailled in [5].

The accuracy of each stochastic formalism (UT/SC) is defined from MC simulations reference. First, it is necessary to determine a reference set of $\langle I\rangle$ values: empirically, the MC convergence appears for $10^{5}$ realizations. Then, in order to compute the error due to stochastic treatments, a criterion is defined standing for this relative error

$$
\operatorname{err}_{i, j}=100 \times \frac{\left|u_{i, j}^{S C / U T / M C}-u_{i, j}^{M C r e f}\right|}{\left|u_{i, j}^{M C r e f}\right|},
$$

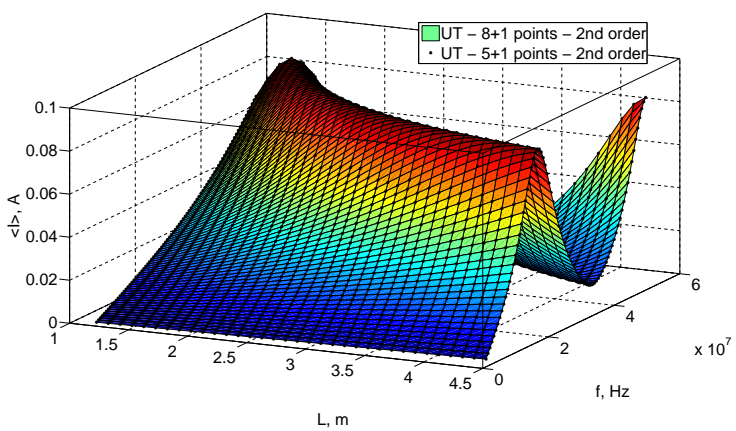

Figure 6: Mean $(I)$ from UT5 and UT8.

considering $\mathrm{err}_{i, j}$ stands for the relative error for each pair $\left(L_{i}, f_{j}\right) \in D_{L} \times D_{f}$. The reference $\mathrm{MC}$ values are given for $10^{6}$ simulations and are depicted previsouly by $u_{i, j}^{M C r e f} . \quad u_{i, j}^{S C / U T / M C}$ represents the elementary $(i, j)$ value obtained respectively from $\mathrm{SC}$, UT and MC (10 ${ }^{5}$ realizations).

The Fig. 7 shows the relative error given for the set of points $\left(L_{k}, f_{k}\right)(k=1, \ldots, N p$ with $N p$ the total number of length/frequency points). The MC convergence may be considered for $10^{5}$ simulations since the error level remains almost everywhere lower than $0.04 \%$ from $10^{6} \mathrm{MC}$ realizations. Obviously, the UT/SC time and memory saves appear clearly from previous example since the stochastic formalisms need less than 10 realizations compared to the $\mathrm{MC}$ technique which requires about 100,000 simulations. In comparison with those MC simulations, the UT/SC curves fit very well and sometimes better precisions are obtained. This perfectly justifies viewing UT/SC as a smart way to proceed to MC. Moreover, for a comparative number of simulations (SC3/UT8 need exactly 9 realizations), the SC method reveals more accurrate than the UT model. Of course, it is possible to improve the UT efficiency by a good choice of the sigma points and weights (and reducing this example to only 6 simulations). The precision levels remain comparable betwen UT and SC but some subdomains show a better accuracy (more than 30 times). The major advantage of SC techniques (high level of accuracy) is clearly illustrated in the Fig. 7.

Furthermore, it would be possible to improve the SC efficiency using techniques from [21] to reduce the number of SC realizations needed, it could be particularly interesting for multivariate stochastic problems involving $4,5, \ldots$ $\mathrm{RV}$ where the SC tensor products lead to some numerical limitations [13]. A numerical problem (and especially a stochastic EMC one) with several RV may appear complex to solve due to the large number of variables. Nevertheless, a solution may be to reduce the number of variables to a minimum regarding their relative influence. Based upon [3], the comparison of results (mean, variance, ...) from one RV simulation with those involving a set of RV provides information on the significant parameters and a view of the model sensitivity.

Finally, the aim of this section was to validate different 


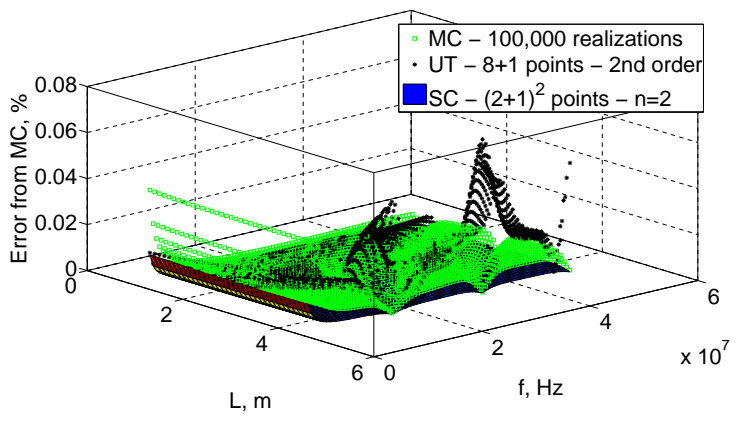

Figure 7: Mean(I) error from reference MC data $\left(10^{6}\right.$ simulations) for MC ( $10^{5}$ realizations $), \mathrm{UT} 8$ and SC3.

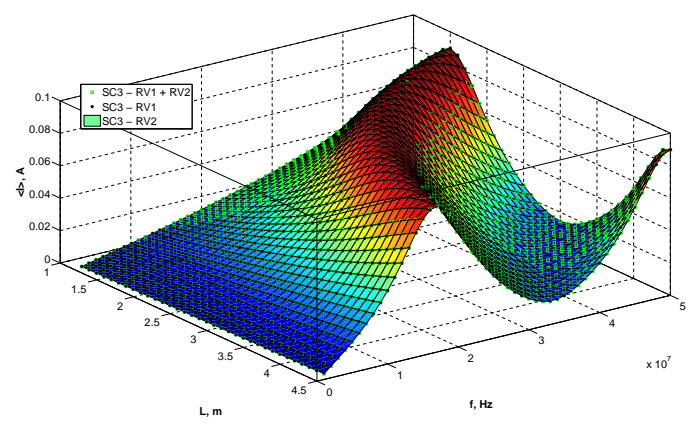

Figure 8: $\langle I\rangle$ from $R V_{1}$ and/or $R V_{2}$.

stochastic techniques (UT/SC) facing results with MC ones. The following part will be dedicated to quantifying the sensitivity influence of different random parameters from the SC method. As depicted previously, without any loss of generality, the same kind of work could be achieved from UT techniques since the UT and SC accuracy were quite similar.

\section{Sensitivity and stochastic studies}

\subsection{A preliminary sensitivity analysis}

In this part, we will put the focus on the use of the SC method in order to lead preliminary sensitivity analysis. From previous definition of the multi-RV issue, we may wonder if the straightforward computing of first statistical moments provides information about the relative sensitivity of RV. Thus, arbitrarily choosing $L$ and $f$ parameters, the Fig. 8 shows that $\langle I\rangle$ including RV1 and/or RV2 as random parameters may not allow to distinguish the most influential parameter from the previous $2 \mathrm{RV}$-set. Indeed, very few differences appear between stochastic treatments involving independently RV1, RV2 and combining $L$ and $f$ parameters.

Obviously, similarly to [3], the variance computing from Tab. 2 may provide more reliable results to distinguish properly the most influential parameters. Indeed, regarding the Fig. 9, differences exist between the three characteristic random treatments involving respectively RV1, RV2 and

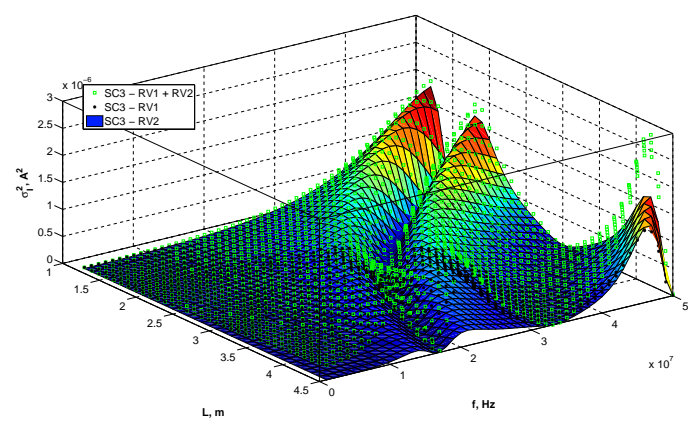

Figure 9: $\sigma_{I}^{2}$ from $R V_{1}$ and/or $R V_{2}$.

RV1+RV2. Obviously, it is possible to better quantify the influence of each parameter from "relative" error criteria considering the 2-RV problem as a reference. However one issue remains particularly constraining since this work is based upon a rough hypothesis assuming the most relevant parameters are well known. That is the reason why, we will detail, in the following paragraph, how the "classical" SA Morris method may offer a reliable and efficient solution.

\subsection{Most influential subset of RV from $\mathrm{SA}$}

From previous theoretical aspects and relation (21), the "Morris" influence criterion $c_{i}$ (13) may be derived from various expressions since the $\chi_{i}$ normalization coefficient has not been defined yet. In the following, we will put the focus on $\chi_{i}$ equivalent to the $i t h$ range length

$$
\chi_{i}=u_{i}^{\max }-u_{i}^{\min },
$$

where $\left[u_{i}^{\min } ; u_{i}^{\max }\right]$ stands for the range of $i t h \mathrm{RV}$.

Considering the relation (18), a screening design model [16] enables us to arrange the set of parameters (Fig. 10) by influence. The model parameters are set to $p=100$ sampling levels, $n=100$ starting points (i.e. 100 uvectors) and $N=100$ pairs $\left(\mu_{i}, \sigma_{i}\right)$ in influence clouds ( $i=1, \ldots, k ; k=12 \mathrm{RV})$. Thus, the impact of the $f$ and $L$ parameters appear quite similar (relatively most influential ones) and some dependency exists between them (physically understandable). Their effect should not be neglected, contrary to most parameters which appear negligible. As expected from (18), the linear "coupling" current $I_{p}$ may behave linearly to other variables and we may set it apart.

From the Fig. 10, we may conclude that our numerical problem with several random parameters may be characterized by a smaller subset of $2 \mathrm{RV}$ : stimulation frequency $f$ and line length $L$.

The SA technique required $n \times(k+1) \times N=130,000$ calls to the analytical formula (18). The global stochastic collocation cost may be evaluated ensuring the convergence of the method (SC3 and SC5), and $50=\left(3^{2}+3 * 2\right)+\left(5^{2}+\right.$ $5 * 2$ ) calls were necessary. Those 130,050 realizations may be compared to the $10^{6} \mathrm{MC}$ simulations needed considering the two previous RV $f$ and $L$. A straightforward $\mathrm{SC}$ approach would have revealed useless since $3^{12}+5^{12}$ 


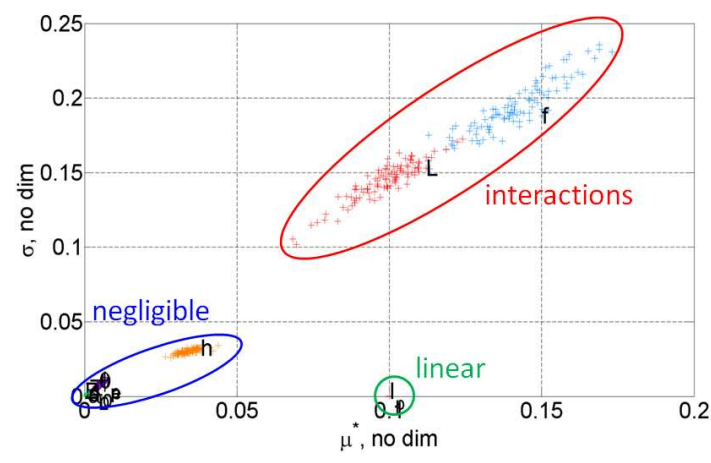

Figure 10: RV ranging from screening design.

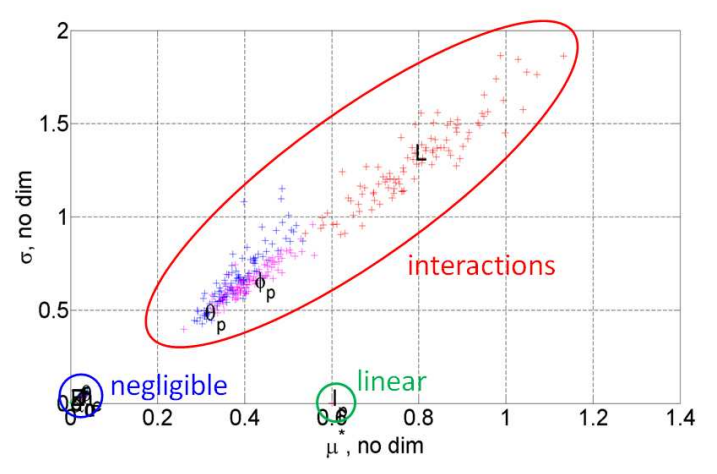

Figure 11: RV ranging from screening design based upon Tab. 4.

calls to the relation (18) would be far less efficient than MC approach. Moreover, the sensitivity study proposed enables lightening the post-treatments since the most influential variables are highlighted as explained in the following.

The SA technique is based upon the initial problem defined. Thus, a different definition of initial RV ranges of variation leads to a distinct issue and involves different results. In this context, we may wonder the quantitative influence effects due to moving initial values from Tab. 3 to Tab. 4.

Table 4: A different set of initial ranges for RV (Fig. 11).

\begin{tabular}{ccccccc}
\hline Value & $L(\mathrm{~m})$ & $h(\mathrm{~mm})$ & $d(\mathrm{~mm})$ & $Z_{L}(\Omega)$ & $E_{0}(\mathrm{~V} / \mathrm{m})$ & $\alpha$ \\
\hline Min & 2.0 & 8 & 0.6 & 900 & 996 & 0. \\
Max & 4.0 & 32 & 1.4 & 1100 & 1004 & 0.01 \\
\hline \hline Value & $Z_{0}(\Omega)$ & $f(\mathrm{MHz})$ & $\theta_{p}(\mathrm{rad})$ & $\theta_{e}(\mathrm{rad})$ & $\phi_{p}(\mathrm{rad})$ & $I_{p}(\mathrm{~A})$ \\
\hline Min & 46 & 890 & 0.0 & 0.0 & 0.0 & 0.2 \\
Max & 54 & 910 & 1.5 & 1.5 & 1.5 & 0.8
\end{tabular}

As depicted in Fig. 11, a distinct set of initial parameters ranges will lead to different conlusions from screening design (most influential parameters: incidence direction through $\theta_{p}$ and $\phi_{p}$ angles, and length $L$ ). Thus, the definition domain of the $\mathbf{u}$ random vector appears as a crucial point.

As explained previously, the aim of this part was to highlight the potentially most influential parameters from a sensitivity analysis. In order to propose a result physically

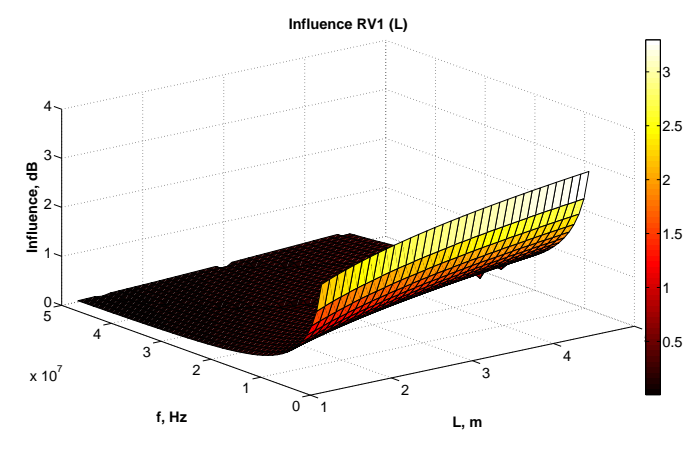

Figure 12: Influence of $L$ parameter (RV1).

understandable, we gave a classical physical interpretation from a well-known global transmission line example. Despite this, the Fig. 11 shows the high impact of the levels of the initial values regarding the sensitivity analysis. Due to the complexity of the problem, different sets of influential parameters may be underlined and the solution is not as straightforward as the common sense view. Based upon initial parameters from Tab. 3, the following section aims to validate quantitatively the result from the qualitative Morris ranking (Fig. 10).

\subsection{Quantitative impact of $R V$}

This part illustrates the SC ability to achieve a reliable sensitivity analysis in a random EMC problem. Among all the variables depicted in Fig. 3, we will focus on the line length and frequency excitation. Based upon previous SA, the $L$ and $f \mathrm{RV}$ need to be included in the stochastic model (other variables may be set to determinsitic mean values). We may rely on the stochastic techniques (SC and/or UT) to lead an entire sensitivity analysis to quantify the impact of one parameter to another.

An influence criterion was defined in [3] to characterize the sensibility of one RV. Based upon the SC results, a similar parameter is

$$
\operatorname{In}_{Z_{i}}=-\log \left(\left|1-\frac{\operatorname{var}\left(I\left(Z_{i}\right)\right)}{\operatorname{var}\left(I\left(Z_{1}, Z_{2}, \ldots, Z_{k}\right)\right)}\right|\right),
$$

with $\operatorname{var}\left(I\left(Z_{i}\right)\right)$ and $\operatorname{var}\left(I\left(Z_{1}, Z_{2}, \ldots, Z_{k}\right)\right)$ the variances of the current $I$ given respectively from one RV $Z_{i}$ $(i=1, \ldots, k)$ and $k$ RV. As depicted previously, the two random outputs are given considering two $\mathrm{RV}$, respectively $R V 1$ for $L$ and $R V 2$ for $f$.

The influence of each parameter is shown in Fig. 12 and Fig. 13. As expected, the relative behaviour of each parameter appears similar since the two parameters are dependent on a physical point of view. The computation of the influence from (22) lays emphasis on the dominant effect of length at low frequency (influence levels greater than $1 \mathrm{~dB}$ ). In comparison with $f$-influence (Fig. 13), the maximum levels are greater regarding $L$-effect ( $I n<1.4 \mathrm{~dB}$ for the frequency). 


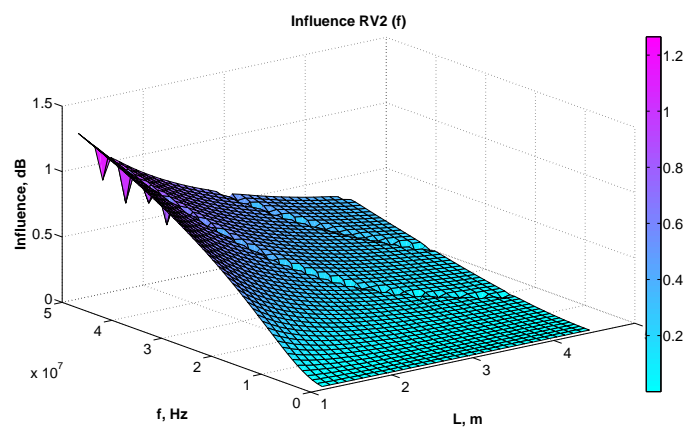

Figure 13: Influence of $f$ parameter (RV2).

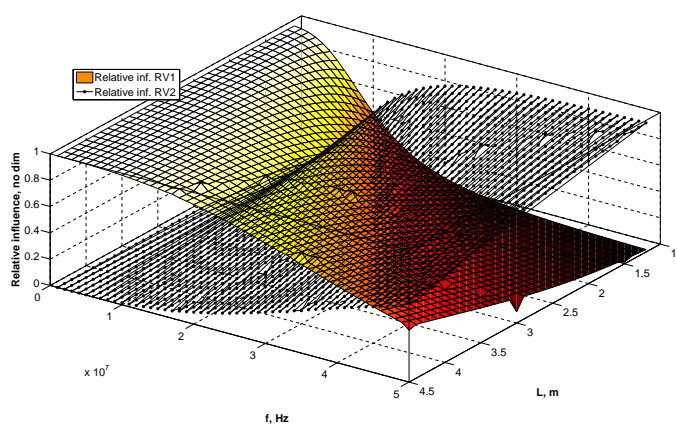

Figure 14: Relative influence of $L / f$ parameter from one to another.

In order to better understand the relative effect of each parameter, we may define a relative influence criterion from previous $I n_{Z_{1}}$ and $I n_{Z_{2}}$ parameters (respectively for $L$ $R V 1$ and $f-R V 2$ ) following

$$
\tilde{I n_{Z_{i}}}=\frac{I n_{Z_{i}}}{\sum_{j=1}^{k} I n_{Z_{j}}}
$$

The Fig. 14 gives an overview of the relative influence of parameters $L$ and $f$ following the relation (23). As expected, the physical dependence between the two random parameters involves a well-balanced distribution between $L$ and $f$. Indeed, based upon the given domain sampling, a quick counting distinguishing major effects of length or frequency shows an influence rate respectively of $55.64 \%$ and $44.36 \%$ for $L$ and $f$. Obviously, this last remark might have been expected from Fig. 10 since the two parameters representation (interaction clouds) were closed. Nevertheless, no quantitative information might be extracted since the "Morris" SA only provides "qualitative" results.

\subsection{Influence of an additional RV}

From the Fig. 10, in the previous section, we laid emphasis on two random parameters: the line length $L$ (geometry) and the stimulation frequency $f$ (disturbing source). Indeed, $L$ and $f$ appeared clearly in Fig. 10 as the most influential variables and needed to be defined in the stochastic model. One parameter was considered as negligible: the height from the ground plane $h$. In this part, we would like to check the validity of our previous SA assessment. Thus, $h$ is quantitatively included in our stochastic issue.

Based upon the relation (22), a third influence criterion may be defined for $h$ with a new RV ( $R V 3$ ). $R V 3$ may be given as depicted in (1) with $\hat{u}_{3}$ following a normal distribution with a variance $\sigma_{\hat{u}_{3}}^{2}=2 \cdot 083 \cdot 10^{-10}\left(\mathrm{~m}^{2}\right)$. Consequently, we may write

$$
h=h^{0}+\hat{u}_{3},
$$

with $h^{0}$ the height mean with $h^{0} \in D_{h}=$ $[8 \mathrm{~mm} ; 32 \mathrm{~mm}]$ according to Tab. 3. Without any loss of generality, different statistical distributions may be defined for $\hat{u}_{1}, \hat{u}_{2}$ and $\hat{u}_{3}$. In this paper, these RV only differ from their variances in accordance with their varying ranges.

In order to simplify the display of results, in this 3-RV study, we propose to represent data by pairs (regarding each time two RV among $L, f$ and $h$ ). Thus, the criteria from the relations (22) and (23) were slightly modified by considering an averaged value over one of the three RV

$$
I n_{Z_{i}}^{j}=\left\langle I n_{Z_{i}}\right\rangle_{j},
$$

with $\langle.\rangle_{j}$ standing for the mean value of $I n_{Z_{i}}$ for $Z_{j} \in$ $D_{Z_{j}}(j=1, \ldots, k)$.

Comparatively to (25) and relying on the relation (23), we may define

$$
\tilde{I n}_{Z_{i}}^{j}=\left\langle\tilde{I n}_{Z_{i}}\right\rangle_{j}
$$

where $\tilde{I n}_{Z_{i}}^{j}$ represents a mean value over $Z_{j}$-dimension of the $Z_{i}$ parameter relative influence $(i, j=1, \ldots, k$; in this part $k=3$ ).

As expected, the parameters $L$ and $f$ remain the most influential from a quantitative point of view. Although the results presented in Fig. 15 are not fully displayed (considering mean values over $h$ and a representation over $D_{L} \times D_{f}$ domain), the major influence of the line length and source frequency appears obvious. As explained previously, in order to get a finer and clearer view of the relative impact of each of the $3 \mathrm{RV}$, the Fig. 16, 17 and 18 based upon the relation (26) are given. Considering the three previous figures, the relative influence of $L$ and $f$ values seems huge comparatively to $h$-parameter. Apart regarding the resonance effect in Fig. 16, the impact of height is minor, less than $0.1 d B$ from (25). Moreover, it is possible to distinguish major effects of $L, f$ and $h$ from a quick counting. The respective $L, f$ and $h$ impact rates are about $55.27 \%, 43.91 \%$ and $0.82 \%$ over the whole domain from the relation (25). This quantitatively justifies the preliminary choice achieved from SA (Fig. 10): the length and frequency remain the most influential parameters.

\section{Conclusion and prospects}

In this contribution, a sensitivity analysis (SA) technique jointly with stochastic methods were presented to solve EMC problems. The use of unscented transform (UT) and 


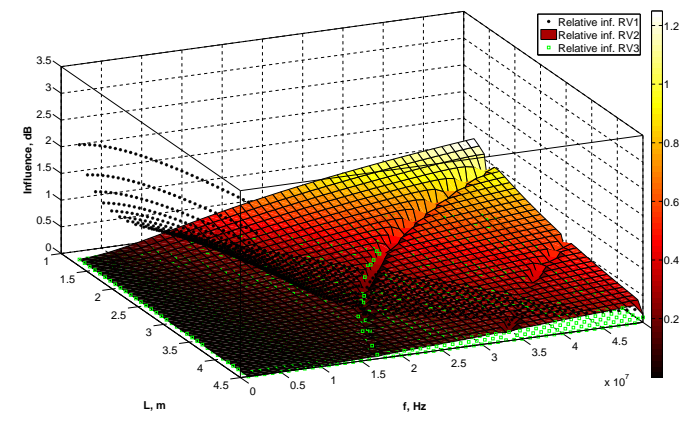

Figure 15: Influence criterion $\operatorname{In}_{Z_{i}}^{3}(i=1,2,3)$.

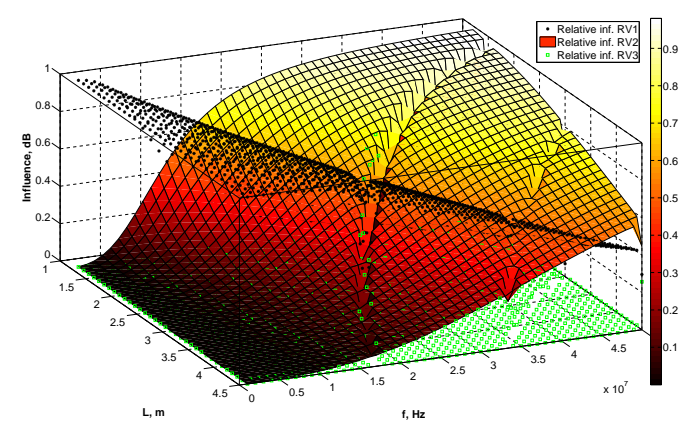

Figure 16: Relative influence criterion $\tilde{I n}_{Z_{i}}^{3}(i=1,2,3)$.

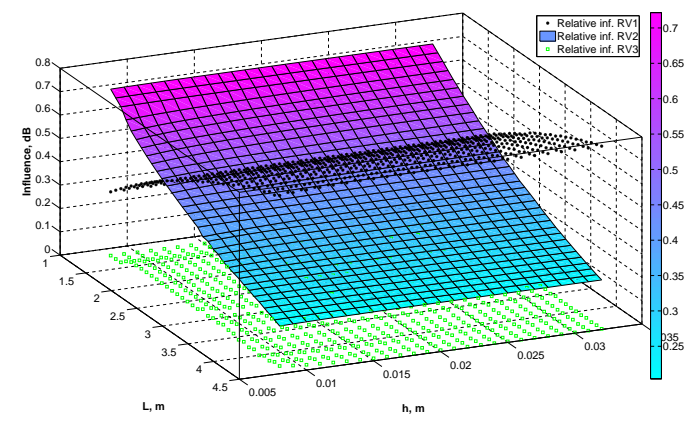

Figure 17: Relative influence criterion $\tilde{I n}_{Z_{i}}^{2}(i=1,2,3)$.

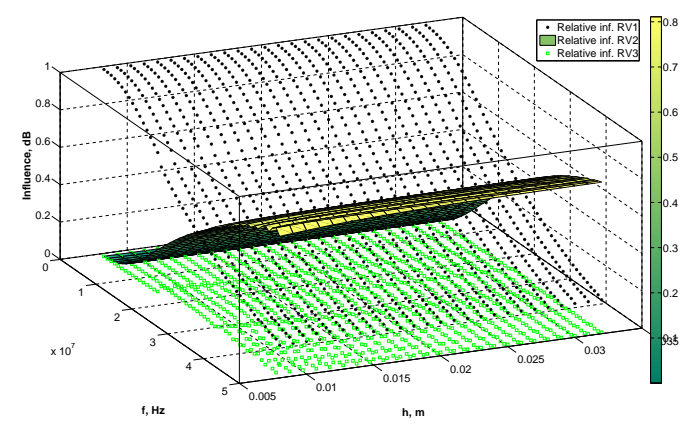

Figure 18: Relative influence criterion $\tilde{I n}_{Z_{i}}^{1}(i=1,2,3)$. stochastic collocation (SC) have been fully justified from their high simplicity and accuracy. Uncertainties involving source parameters (frequency) and geometry of a transmission line (length) have been defined considering various random variables (RV) UT/SC efficiency and precision were detailled regarding this stochastic EMC issue in multi-RV case. Since the UT and SC methods are similar to well chosen MC simulations, their efficiency appears to be an important advantage compared with Monte Carlo (MC): minimizing computing time more than 20,000 times. Moreover, one of their main advantages relies on their non-intrusive characteristic: similarly to MC, the techniques only need to achieve numerical measurements for particular input parameter values (without causing deep alteration in electromagnetic codes). Unfortunately, the efficiency may drastically decrease when the number of RV increases. That's the reason why we proposed a SA method to determine what are the most important variables. Based upon SA results, only significant parameters were studied to quantitatively compute their influence domains. This may be particullarly interesting to optimize EMI/EMC tests and to improve EMC equipments design. The whole process may perfectly apply oneself to other electromagnetic simulation/experimental tools. Recent studies are nowadays in progress in order to validate the use of stochastic techniques in an experimental EM complex environment [22, 23].

\section{References}

[1] W.H. Press, S.A. Teukolsky, W.T. Vetterling, B.P. Flannery, Numerical Recipes, Cambridge University Press, 2nd edition, 1992.

[2] N. Mishra, N. Gupta, Quasi Monte Carlo Integration Technique for Method of Moments Solution of EFIE in Radiation Problems, ACES Journal, vol. 24, no. 3, pp. 306-311, June 2009.

[3] L. de Menezes, D. Thomas, C. Christopoulos, Accounting for uncertainty in EMC studies, Proc. 2009 Int. Symp. on EMC, Kyoto, Japan, 2009.

[4] L. de Menezes, D. Thomas, C. Christopoulos, A. Ajayi, P. Sewell, The use of unscented transforms for statistical analysis in EM, Proc. EMC Europe 2008, Hamburg, Germany, 2008.

[5] L. de Menezes, A. Ajayi, C. Christopoulos, P. Sewell, G.A. Borges, Efficient computation of stochastic electromagnetic problems using unscented transforms, IET Sci. Meas. Technol., vol. 2(2), pp. 88-95, 2008.

[6] C. Chauvière, J.S. Hestaven, L.C. Wilcox, Efficient Computation of RCS from Scatterers of Uncertain Shapes, IEEE Trans. On Ant. And Prop., vol. 55, no. 5, pp. 1437-1448, May 2007.

[7] P. Bonnet, F. Diouf, C. Chauvière, S. Lalléchère, M. Fogli, F. Paladian, Numerical simulation of a Reverberation Chamber with a stochastic collocation 
method, Compte Rendu de l'Académie des Sciences, Physique, vol. 10, pp. 54-64, 2009.

[8] P. Bonnet, C. Chauvière, S. Lalléchère, F. Paladian, B. Pecqueux, Recherche de configurations critiques pour un problème de C.E.M. stochastique, Proc. 15th Int. Symp. on EMC, Limoges, France, April 2010.

[9] F. Paladian, P. Bonnet, S. Lalléchère, Modeling complex systems for EMC applications by considering uncertainties, Proc. XXXth URSI GA 2011, Istambul, Turkey, August 2011.

[10] F. Diouf, F. Canavero, Crosstalk statistics via collocation method, Proc. IEEE Int. Symp. on Electromagnetic Compatibility, Austin TX, USA, Aug. 2009.

[11] V. Rannou, F. Brouaye, M. Hélier, W. Tabbara, Kriging the quantile: application to a simple transmission line model, Inst. of Ph. Publish., Inverse problems 18, pp. 37-48, PII: S0266-5611(02)20767-4.

[12] A. El Habachi, Propagation de la variabilité de la morphologie humaine sur le Débit d'Absorption Spécifique en dosimétrie numérique, PhD Thesis Supélec, Paris, France, 2011.

[13] H. Bagci, A.C. Yucel, J.S. Hesthaven, E. Michielssen, A fast stroud-based collocation method for statistically characterizing EMI/EMC phenomena on complex platforms, IEEE Trans. on EMC, vol. 51, no. 2, pp. 301-311, 2009.

[14] P.S. Sumant, H. Wu, A.C. Cangellaris, N.R. Aluru, A sparse grid based collocation method for model order reduction of finite element approximations of passive electromagnetic devices under uncertainty, Proc. 2010 IEEE MTT-S International, Anaheim, CA, USA, pp. 1652-1655, May 2010.

[15] I. Tarhini, C. Guiffaut, A. Reineix, S. Karam, B. Pécqueux, J.C. Joly, Etude paramétrique de la susceptibilité des cartes électroniques par les plans d'expériences numériques, Proc. Int. Symp. On EMC, Paris, France, May 2008.

[16] M.D. Morris, Factorial sampling plans for preliminary computational experiments, Technometrics, Vol. 33, No. 2, pp. 161-174, 1991.

[17] A. Saltelli, M. Ratto, S. Tarantola, F. Campolongo, Sensitivity analysis practices. Strategies for modelbased inference, Reliability Engineering and System Safety, vol. 91, pp. 1109-1125, 2006.

[18] B. Iooss, Revue sur l'analyse de sensibilité globale de modèles numériques, Journal de la Société Française de Statistiques, vol. 152, no. 1, pp. 3-25, 2011.

[19] F. Campolongo, J. Cariboni, A. Saltelli, V. Schoutens, Enhancing the Morris method, Sensitivity Analysis of Model Output, Los Alamos National Laboratory, 2005.
[20] D. Xiu, J.S. Hesthaven, High order collocation methods for differential equations with random inputs, SIAM J. Sci. Comput., Vol. 27, No. 3, pp. 1118-1139, 2005.

[21] A.H. Stroud, Remarks on the disposition of points in numerical integration formulas, Math. Tables Other Aids Comput., vol. 11, no. 3, pp. 1118-1139, Oct. 1957.

[22] D.W.P. Thomas, J. Paul, C. Christopoulos, Statistical variation of coupling within a circuit cabinet, Proc. ESA workshop on aerospace EMC, Venice, Italy, May 2012.

[23] S. Lalléchère, S. Girard, P. Bonnet, F. Paladian, Enforcing experimentally stochastic techniques in uncertain electromagnetic environments, Proc. ESA workshop on aerospace EMC, Venice, Italy, May 2012. 